\title{
Use of Electronic Patient Reported Outcomes and Automated Devices for Heart Failure Disease Management
}

Sean Pinney; Emamuzo Otobo; Robert Freeman; Jason Rogers; Farah Fasihuddin; Karthik Ramireddy; Allyssa Deorocki; Neda Hassanzadeh; Marni Goldstein; Rivelle Zlatopolsky; Jennifer Ullman; Ashish Atreja

Icahn School of Medicine at Mount Sinai, New York, NY, United States

\author{
Corresponding Author: \\ Sean Pinney \\ Icahn School of Medicine at Mount Sinai \\ 1468 Madison Avenue \\ New York, NY, \\ United States \\ Phone: 12416500 \\ Email: sean.pinney@mssm.edu
}

\begin{abstract}
Background: Congestive heart failure (CHF) is a major public health issue. Today, CHF affects 6.5 million people in the U.S. and the incidence rate is projected to rise by $46 \%$ to more than 8 million cases by 2030 . Current reimbursement policies use readmission rates and length of hospital stays as indicators of quality of care, and incentivize providers to meet these quality measures as the cost of hospitalization alone significantly contributes to the overall burden of CHF on patients and health systems. Symptoms of CHF can be unpredictable and presently there are no reliable solutions to track disease control for discharged patients.
\end{abstract}

Objective: This study aims to improve patients' self-monitoring practices post-hospital discharge, quickly identify critical warning signs, decrease hospital readmissions and reduce healthcare costs for CHF patients by integrating remote monitoring ePRO into standard outpatient care practices.

Methods: The pilot study seeks to enroll 60 patients who were admitted to The Mount Sinai Hospital for acute CHF exacerbation and have access to either an Android or iOS enabled smartphone. RxUniverse (a digital platform that enables physicians to directly "prescribe" evidence-based mobile health applications to patients) will be used to prescribe HealthPROMISE and iHealth mobile apps (Figure 1). Patients update and record their CHF-related symptoms and quality of life measures daily on HealthPROMISE. Vital sign data, including blood pressure, heart rate, and weight, is collected through an ambulatory remote monitoring system that includes a smartphone application and Bluetooth-connected smart devices. ePRO data is submitted electronically to a dashboard monitored daily by a practitioner, who determines whether to continue current care or to call the patient for further assessment of symptoms (Figure 2). Any critical red-flag values automatically alert the physician and prompt the patient to seek medical attention. Enrollment barriers included: onboarding time (30 minutes), identifying patients, competition with other Mount Sinai initiatives and research trials, language barriers, and low health literacy (Figure 3).

Results: The study had 52 of the 60 patients enrolled. Thirty-eight patients (73\%) continue to actively use the mobile apps and smart devices to track blood pressure and weight, 27 patients (52\%) have completed one month of active use, while 5 patients have dropped out. There have been 4 hospital readmissions (7\%) mainly due to non-compliance and complications from other chronic conditions (Figure 3).

Conclusions: Given the increasing burden of $\mathrm{CHF}$ on patients and healthcare systems, there is a critical need for an effective, sustainable, and feasible remote monitoring system for CHF patients following hospital discharge. The ability for providers to access patient-reported outcomes and vital signs in real-time can significantly impact the quality of outpatient care, potentially reducing readmissions and costs. CHF patients are showing positive health outcomes; CHF patients had a 7\% readmission rate compared to the national readmission rate of $>25 \%$ within 30 days of discharge. Enrollment challenges were overcome by enrolling CHF patients 2-3 days before expected discharge and adding a patient coordinator to hospital rounds. These latest advances in remote monitoring show promise for the future of technology-connected healthcare.

(iproc 2017;3(1):e24) doi: 10.2196/iproc.8459 


\section{KEYWORDS}

congestive heart failure; sensors; quality of life; electronic patient reported outcomes; remote monitoring; self-management; hospital readmission

\section{Figures}

Figure 1. CHF Solution tool kit, including RxUniverse (used to "prescribe" patients the apps), HealthPROMISE (used for patients to track symptoms), and iHealth (used to track data from the smart devices).

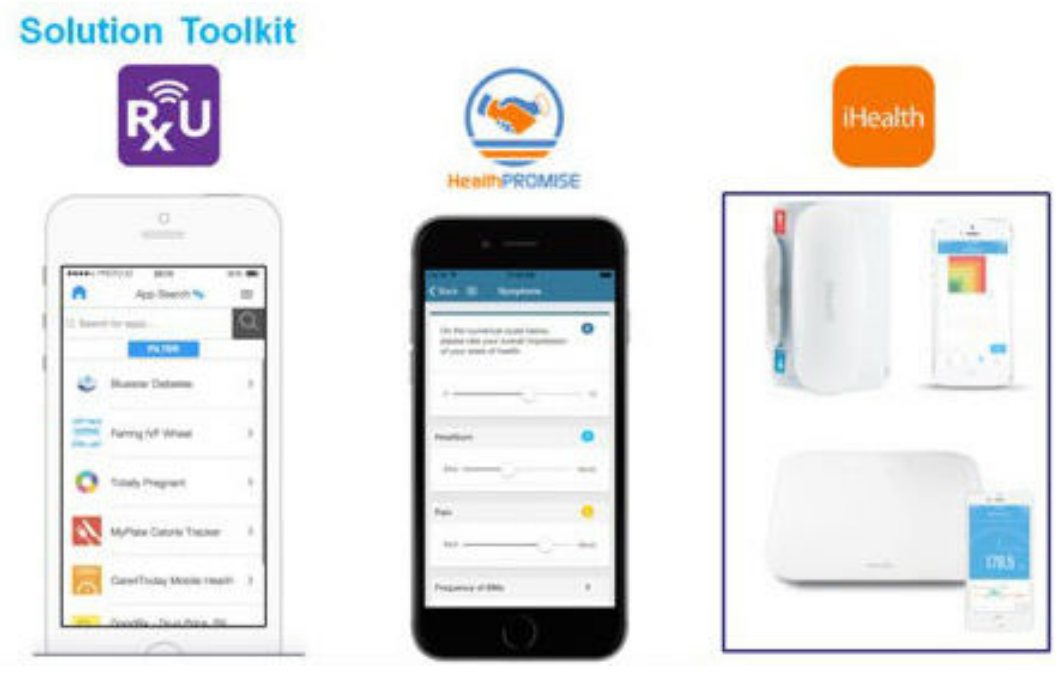

Figure 2. Vital sign measurements in real time transmitted to provider dashboard.

\section{Real time connected device data: Blood Pressure and weight}

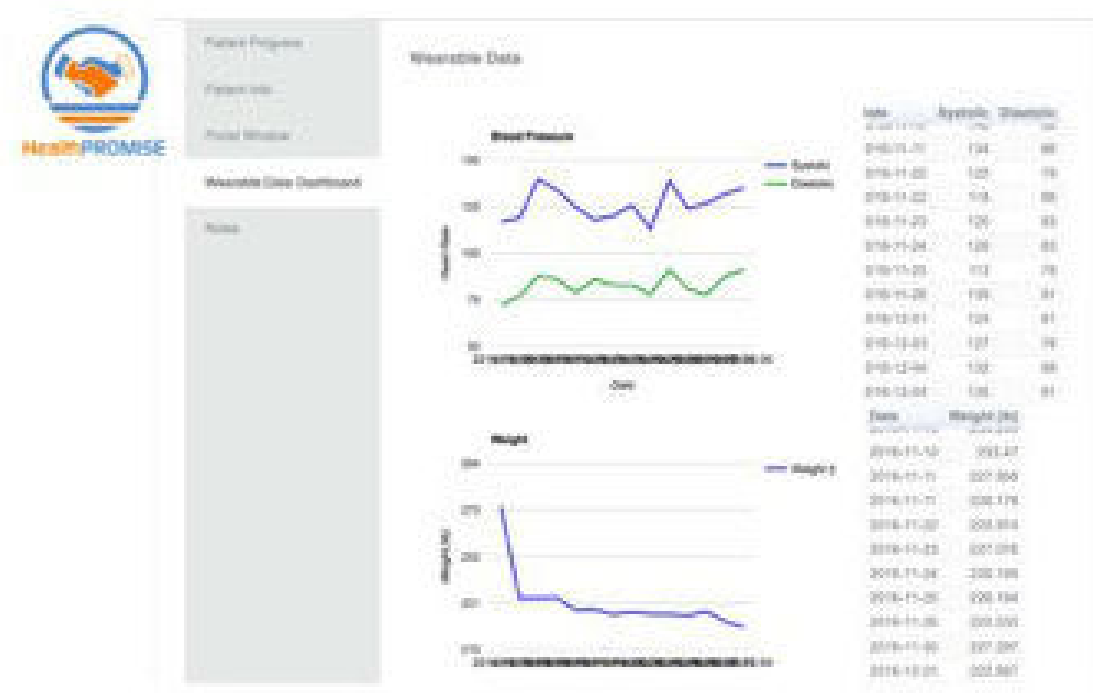


Figure 3. Enrollment flow chart.

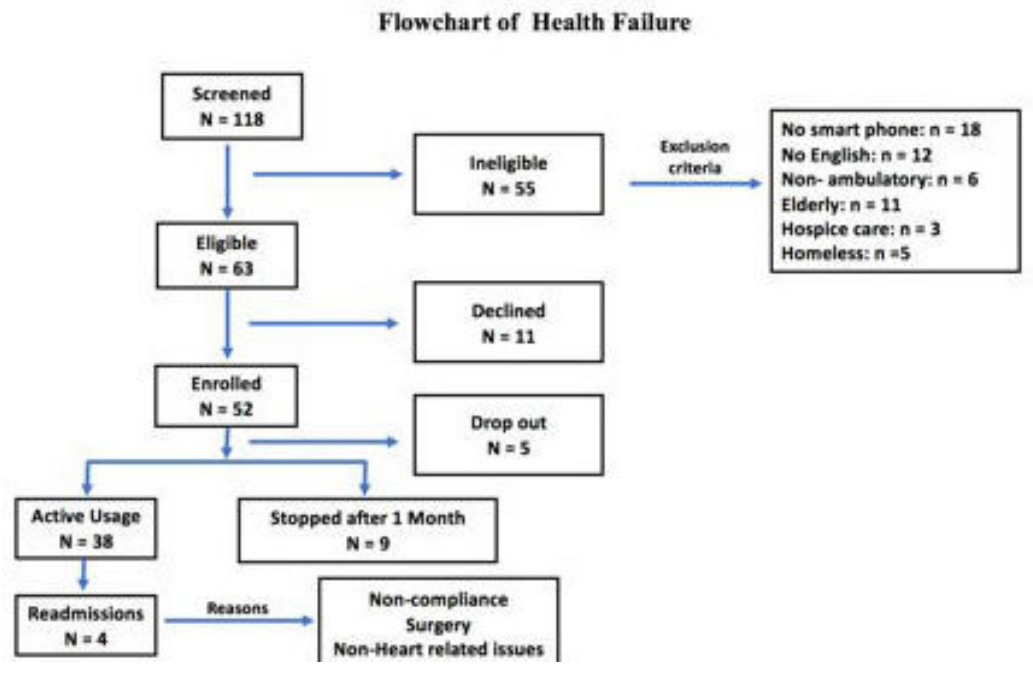

\section{Multimedia Appendix 1}

Full poster.

[PDF File (Adobe PDF File), 1MB-Multimedia Appendix 1]

Edited by T Hale; this is a non-peer-reviewed article. Submitted 13.07.17; accepted 24.08.17; published 22.09.17.

Please cite as:

Pinney S, Otobo E, Freeman R, Rogers J, Fasihuddin F, Ramireddy K, Deorocki A, Hassanzadeh N, Goldstein M, Zlatopolsky R, Ullman J, Atreja A

Use of Electronic Patient Reported Outcomes and Automated Devices for Heart Failure Disease Management

iproc 2017;3(1):e24

URL: http://www.iproc.org/2017/1/e24/

doi: 10.2196/iproc. 8459

PMID:

(CSean Pinney, Emamuzo Otobo, Robert Freeman, Jason Rogers, Farah Fasihuddin, Karthik Ramireddy, Allyssa Deorocki, Neda Hassanzadeh, Marni Goldstein, Rivelle Zlatopolsky, Jennifer Ullman, Ashish Atreja. Originally published in Iproceedings (http://www.iproc.org), 22.09.2017. This is an open-access article distributed under the terms of the Creative Commons Attribution License (https://creativecommons.org/licenses/by/4.0/), which permits unrestricted use, distribution, and reproduction in any medium, provided the original work, first published in Iproceedings, is properly cited. The complete bibliographic information, a link to the original publication on http://www.iproc.org/, as well as this copyright and license information must be included. 